\title{
Clinical outcomes of three- or five-day treatment with clomiphene citrate combined with gonadotropins and a timed intercourse cycle in polycystic ovary syndrome patients
}

\author{
Sung-Ah Bae ${ }^{1 *}$, Jong-Kil Joo ${ }^{2 *}$, Jong-Ryeol Choi ${ }^{3}$, Sun-Suk Kim² ${ }^{2}$ Kyu-Sup Lee ${ }^{2}$ \\ 'Pusan National University Medical Graduate School, Yangsan; ${ }^{2}$ Department of Obstetrics and Gynecology, Medical Research Institute, Pusan National \\ University School of Medicine, Yangsan; ${ }^{3}$ Eroom Women's Clinic, Busan, Korea
}

Objective: This study aimed to investigate the effect of a new clomiphene citrate (CC) regimen on preventing thin endometrial lining in polycystic ovary syndrome (PCOS) patients receiving CC plus gonadotropin treatment with a timed intercourse cycle.

Methods: A total of 114 women with PCOS were included in this trial. Patients were divided into two groups and treated in accordance with the controlled ovarian stimulation (COS) protocol. In group A, 104 COS cycles in 67 patients were included, and in each cycle $150 \mathrm{mg}$ CC was given for three days, starting from day 3. In group B, 69 COS cycles in 47 patients were included, in which $100 \mathrm{mg}$ CC was given for five days, starting from day 3 . The thickness of the endometrium was measured on the day of human chorionic gonadotropin (hCG) injection. Timed intercourse was recommended at 24 and 48 hours after the $\mathrm{hCG}$ injection.

Results: Additional doses of human menopausal gonadotropin and the number of days of hCG administration were not significantly different between the two groups. Endometrial thickness on the day of hCG administration was significantly larger in group A than group B $(9.4 \pm 2.1$ $\mathrm{mm}$ vs. $8.5 \pm 1.7 \mathrm{~mm}, p=0.004)$. The pregnancy rate was significantly higher in group $A$ than in group $B(38.4 \%$ vs. $21.7 \%, p=0.030)$.

Conclusion: Three-day $C C$ treatment resulted in a significantly higher pregnancy rate than the standard five-day CC treatment in a timed intercourse cycle in PCOS patients. Facilitating adequate endometrial growth via the early discontinuation of CC might be a crucial factor in achieving a higher pregnancy rate.

Keywords: Clomiphene citrate; Endometrium; Pregnancy; Timed intercourse

\section{Introduction}

Clomiphene citrate (CC) is used for the induction of ovulation or

Received: Apr 6, 2015 · Revised: Jun 17, 2015 · Accepted: Jul 6, 2015 Corresponding author: Kyu-Sup Lee

Department of Obstetrics and Gynecology, Medical Research Institute, Pusan National University School of Medicine, 49 Busandaehak-ro, Mulgeum-eup, Yangsan 50612, Korea

Tel: +82-51-240-7287 Fax:+82-51-248-2384 E-mail:kuslee@pusan.ac.kr

*The first two authors contributed equally to this work.

*This work was supported by a clinical research grant from Pusan National University Hospital in 2015.

This is an Open Access article distributed under the terms of the Creative Commons Attribution Non-Commercial License (http://creativecommons.org/licenses/by-nc/3.0/) which permits unrestricted non-commercial use, distribution, and reproduction in any medium, provided the original work is properly cited. growth of multiple follicles in the treatment of infertility. It binds to estrogen receptors in the hypothalamus and this binding causes an anti-estrogenic effect. By a negative feedback mechanism, CC stimulates the gonadotropin-releasing hormone pulse and induces gonadotropin secretion from the anterior pituitary gland [1,2].

CC is considered to be an inexpensive, effective, and safe drug for ovulation induction. For these reasons, it is widely used as a first-line treatment for anovulatory and unexplained infertility. CC induces ovulation in approximately $70 \%$ to $85 \%$ of patients, but the pregnancy rate is lower than the ovulation rate. This may be due to the antiestrogenic effects of CC on endometrial growth and cervical mucus production, a decrease in uterine blood flow, impaired synthesis of placental protein 14 , subclinical pregnancy loss, impaired tubal trans- 
port, and detrimental effects on oocytes [3]. Of these, decreased endometrial development is a major undesired effect [4-6]. In fact, endometrial thickness is significantly thinner in women taking CC than in women not taking CC [4-6]. Satirapod et al. [7] reported an endometrial thickness of around $9.04 \mathrm{~mm}$ in women taking CC, while endometrial thickness in a natural cycle group was about $9.52 \mathrm{~mm}$ on the day of ovulation. Thus, overcoming the anti-estrogenic effect of $\mathrm{CC}$ on endometrial growth is important for achieving pregnancy in CC-assisted cycles. The anti-estrogenic effect of CC is exerted in a dose-dependent manner and continues until the late follicular phase in the standard CC treatment cycle. Therefore, using different doses and a short duration of CC treatment might be a solution for improving endometrial growth during the CC cycle.

In this study, we compared endometrial growth and pregnancy rates between two different protocols: $150 \mathrm{mg}$ CC for three days and $100 \mathrm{mg}$ CC for five days, with additional gonadotropins, in a time intercourse cycle in polycystic ovary syndrome (PCOS) patients.

\section{Methods}

This study was a retrospective pilot study conducted by a university and a private infertility clinic. A total of 114 women aged less than 40 years were included in the study from January 2013 to March 2014. Patients conducting timed intercourse, but not intrauterine sperm injection (IUI), were collected consecutively without omission. The inclusion criteria for the patients were as follows: (1) a basal folliclestimulating hormone (FSH) level of less than $10 \mathrm{mlU} / \mathrm{mL}$, (2) normal hysterosalpingography (HSG) findings, (3) an adequate number of sperm for timed insemination, and (4) a PCOS diagnosis identified by the 2003 European Society for Human Reproduction/American Society of Reproductive Medicine (ESHRE/ASRM, Rotterdam) criteria.

The patients in this study were non-smokers and did not have any major medical illness including hypertension. Patients were excluded if they had myoma, adenomyosis, a congenital uterine anomaly, or ovarian tumors. Patients were also excluded if they used estrogens, progesterone, androgens, or had a history of chronic use of any medication within the previous three months, including non-steroidal anti-inflammatory agents. None of the patients showed abnormal findings in the uterine cavity on HSG.

Patients were divided into two groups and treated in accordance with the controlled ovarian stimulation (COS) protocol. In group A, 104 COS cycles in 67 patients were included. In each cycle, $150 \mathrm{mg}$ CC (Clomid, Young Poong Corp., Seoul, Korea) was given for three consecutive days starting on the third day of the cycle. In group B, 69 COS cycles in 47 patients were included. $100 \mathrm{mg}$ CC was given for five consecutive days starting on the third day of the cycle. In both groups, additional human menopausal gonadotropin (hMG) Meno- pur (Ferring Pharmaceuticals, Kiel, Germany) was administered every other day; patients weighing more than $60 \mathrm{~kg}$ received $225 \mathrm{IU}$ hMG per dose and those weighing below $60 \mathrm{~kg}$ received $150 \mathrm{IU} \mathrm{hMG}$. hMG administration started on day 7 and continued until human chorionic gonadotropin (hCG) administration, after which the dose of hMG was adjusted according to ovarian response. Endometrial thickness and follicular growth were assessed by vaginal ultrasonography (Voluson S8-5451540, Voluson E8C vaginal transducer, GE Healthcare, Waukesha, WI, USA).

In cases of premature luteinizing hormone (LH) surge or no response, the cycle was cancelled. Premature LH surge was diagnosed by several ultrasonographic characteristics, such as the appearance of a corpus luteum, the disappearance of the triple line of endometrium, and fluid collection at the cul-de-sac. If there was no follicular growth after the fifteenth day of the cycle and no increase in follicular diameter at two separate follow-up ultrasonographic examinations, then there was a diagnosis of 'no response'.

When follicles reached a diameter of $18 \mathrm{~mm}$ as measured by ultrasonography, $500 \mu \mathrm{g}$ chorionic gonadotropin alpha (Ovidrel Pen, Merck Serono, Darmstadt, Germany) was administered to induce ovulation. After a longitudinal view of the uterus was obtained, the thickness of the endometrium was measured at the maximum distance between each endometrial-myometrial interface on the day of hCG administration. The number of follicles on the day of hCG administration was also assessed by vaginal ultrasonography. Timed intercourse was recommended at 24 and 48 hours after hCG injection.

\section{Statistical analysis}

Statistical analysis was carried out with PASW ver. 18.0 (SPSS Inc., Chicago, IL, USA). The Mann-Whitney U test with Bonferroni correction, Fisher's test, and Kruskal-Wallis $\mathrm{H}$ test were employed as appropriate. Differences were considered to be significant if the $p$-value was less than 0.05 . Data were expressed as mean \pm standard deviation.

\section{Results}

Table 1 presents the patients' characteristics. There was no significant difference between the two groups in the age of patients, duration of infertility, or basal hormone levels including FSH, LH, free testosterone, estradiol, prolactin, dehydroepiandrosterone-sulfate, and thyroid stimulating hormone. Body mass index (BMI) was not significantly different between groups.

COS outcomes can be ascertained from Table 2. Two patients in group A showed a premature LH surge and did not follow the timed intercourse cycle. Three other patients had no response until menstrual cycle day 20,22 , and 23 , respectively, and these cycles were 
also cancelled. In group B, no premature LH surge was observed; however, three cycles were cancelled because of a poor response. None of the cancelled cycles were included in the final statistical analysis.

The number of additional hMG doses was not significantly different between the two groups. The number of days of hCG administration and mature follicle counts on the day of hCG administration were similar between both groups. The endometrial thickness on the day of hCG administration was significantly different between the two groups; the endometrial thickness in group A was greater than that in group $B$. The proportion of patients with a thin endometrium ( $<7$ $\mathrm{mm}$ ) on the day of hCG administration was not significantly different between groups.

Clinical outcomes can be ascertained from Table 3. The pregnancy rate was significantly higher in group $A$. The multiple pregnancy rate was similar in both groups. One case of triplet pregnancy was observed in group $A$; all other multiple pregnancies were twin pregnan-

Table 1. Patient characteristics

\begin{tabular}{lrcc}
\hline Characteristic & $\begin{array}{c}\text { Group A } \\
(\mathrm{n}=67)\end{array}$ & $\begin{array}{c}\text { Group B } \\
(\mathrm{n}=47)\end{array}$ & $p$-value \\
\hline Age $(\mathrm{yr})$ & $31.3 \pm 3.9$ & $31.1 \pm 3.4$ & 0.772 \\
Duration of infertility $(\mathrm{yr})$ & $1.5 \pm 0.8$ & $1.5 \pm 0.8$ & 0.785 \\
Body mass index $\left(\mathrm{kg} / \mathrm{m}^{2}\right)$ & $20.9 \pm 2.8$ & $20.8 \pm 3.3$ & 0.858 \\
Basal hormone level & & & \\
FSH $(\mathrm{mlU} / \mathrm{mL})$ & $6.6 \pm 1.8$ & $6.4 \pm 1.5$ & 0.576 \\
LH $(\mathrm{mlU} / \mathrm{mL})$ & $9.5 \pm 5.3$ & $8.2 \pm 4.6$ & 0.164 \\
Free testosterone $(\mathrm{pg} / \mathrm{mL})$ & $1.2 \pm 0.7$ & $1.0 \pm 0.6$ & 0.279 \\
Estradiol $(\mathrm{pg} / \mathrm{mL})$ & $37.9 \pm 19.3$ & $38.1 \pm 17.8$ & 0.937 \\
Prolactin $(\mathrm{ng} / \mathrm{mL})$ & $15.5 \pm 10.8$ & $14.3 \pm 9.6$ & 0.522 \\
DHEA-S $(\mu \mathrm{g} / \mathrm{dL})$ & $226.1 \pm 91.1$ & $224.1 \pm 82.2$ & 0.904 \\
TSH $(\mu \mathrm{lU} / \mathrm{mL})$ & $1.7 \pm 1.1$ & $2.0 \pm 1.2$ & 0.287 \\
\hline
\end{tabular}

Values are presented as mean \pm standard deviation.

FSH, follicle-stimulating hormone; LH, luteinizing hormone; DHEA-S, dehydroepiandrosterone-sulfate; TSH, thyroid stimulating hormone. cies. The abortion rate was similar in both groups. Five cases of mild ovarian hyperstimulation syndrome (OHSS) developed.

\section{Discussion}

It is well known that endometrial growth is one of the most important factors in implantation. Esmailzadeh and Faramarzi [8] reported that endometrial thickness on the day of hCG administration was significantly greater in CC-IUI cycles in which pregnancy was achieved. Furthermore, according to El-Toukhy et al. [9], the lowest pregnancy rates are associated with endometrial thicknesses of $<7$ $\mathrm{mm}$ or $>14 \mathrm{~mm}$ in frozen-thawed embryo replacement cycles.

Dehbashi et al. [4] reported that endometrial thickness was significantly thinner in women taking CC than in a control group during the late proliferative phase. These authors suggested several methods to overcome the problem of a thin endometrium after the use of $\mathrm{CC}$, such as the use of phytoestrogens, transdermal estrogen, or an aromatase inhibitor.

Unfer et al. [10] reported the effectiveness of CC in ovulation induction, either alone or combined with phytoestrogens, in patients receiving intrauterine insemination, stating that a high dose of phytoestrogens might reverse the deleterious effects of CC on endometrial thickness. However, safety data and proper protocols for the use of

Table 3. Clinical outcomes in patients with successful ovulation induction

\begin{tabular}{lccc}
\hline & $\begin{array}{c}\text { Group A } \\
(\mathrm{n}=99)\end{array}$ & $\begin{array}{c}\text { Group B } \\
(\mathrm{n}=66)\end{array}$ & $p$-value \\
\hline Pregnancy rate & $38.4(38)$ & $21.7(15)$ & 0.030 \\
No. of pregnancies after first cycle & 25 & 8 & \\
No. of pregnancies after second cycle & 13 & 7 & \\
Multiple pregnancy rate & $18.4(7)$ & $20.0(3)$ & 0.800 \\
Abortion rate & $15.8(6)$ & $20.0(3)$ & 0.677 \\
\hline
\end{tabular}

Values are presented as percent (number).

Table 2. COS outcomes and endometrial thickness in patients receiving three- or five-day treatment

\begin{tabular}{|c|c|c|c|}
\hline Outcome & $\begin{array}{l}\text { Group A } \\
(n=104)^{a)}\end{array}$ & $\begin{array}{l}\text { Group B } \\
\left.(n=69)^{b}\right)\end{array}$ & $p$-value \\
\hline Cycles cancelled due to premature LH surge or poor response (n) & 5 & 3 & - \\
\hline Cycles ovulated with hCG injection (n) & 99 & 66 & - \\
\hline Total hMG dose (IU) & $698.0 \pm 278.6$ & $715.3 \pm 267.5$ & 0.689 \\
\hline Days of hCG administration & $14.2 \pm 1.9$ & $14.0 \pm 1.8$ & 0.722 \\
\hline Mature follicle ( $\geq 16 \mathrm{~mm}$ ) count on the day of hCG administration $(\mathrm{n})$ & $2.8 \pm 1.7$ & $2.7 \pm 1.1$ & 0.312 \\
\hline Endometrial thickness on the day of hCG administration (mm) & $9.4 \pm 2.1$ & $8.5 \pm 1.7$ & 0.004 \\
\hline Proportion of patients with a thin endometrium ( $<7 \mathrm{~mm}$ ) on the day of $\mathrm{hCG}$ administration (\%) & 8.1 & 15.9 & 0.156 \\
\hline
\end{tabular}

Values are presented as mean \pm standard deviation.

$\mathrm{COS}$, controlled ovarian stimulation; LH, luteinizing hormone; $\mathrm{hCG}$, human chorionic gonadotropin; $\mathrm{hMG}$, human menopausal gonadotropin .

a) 104 COS cycles in 67 patients; ${ }^{\text {b) }} 69$ COS cycles in 47 patients. 
phytoestrogens have not yet been established, and there is a risk that these substances may increase the risk of endometrial hyperplasia.

Transdermal estrogen is also used for endometrial growth in CC treatment cycles [11]. Shimoya et al. [11] reported that endometrial thickness was significantly thicker in patients receiving CC and transdermal estrogen, but did not report the pregnancy rate. There is a risk that administration of additional estrogen might affect LH levels and cause an LH surge at an earlier stage or induce a higher progesterone level.

Recently, aromatase inhibitors have been widely used for ovulation induction in patients with anovulatory or unexplained infertility. Because aromatase inhibitors do not deplete estrogen receptors in central and peripheral target tissues, they may have no negative impact on endometrium [12]. Barroso et al. [13] reported that a significantly thicker endometrium was observed in patients who received letrozole than in patients who received CC $(9.5 \pm 1.5 \mathrm{~mm}$ vs. $7.3 \pm 1.1 \mathrm{~mm}$, $p<0.001)$. However, aromatase inhibitors are relatively expensive and there are still some safety issues with their use [14].

Takasaki et al. [14] conducted a prospective randomized controlled study to examine the effect of either reduced-dose or early administration of CC in patients with a thin endometrium compared with standard CC treatment. They reported that the endometrium was thicker in the two experimental groups than in the standard group. $\mathrm{CC}$ is cleared through the liver and excreted in the stool, and approximately $85 \%$ is eliminated within a week [15]. In this study, we used a protocol of early discontinuation of CC. We found that the endometrium was thicker and the pregnancy rate was higher in the early discontinuation group, while the number of follicles larger than $16 \mathrm{~mm}$ in diameter was not different.

There are some limitations to this study. First, the number of patients included in the study was relatively small and treatment was not randomized. Also, there were differences in the number of patients enrolled in each group that arose in the process of selecting patients according to the criteria of each group. Second, this study included only PCOS patients, who are supposed to be high responders to the treatment used, and the dose of CC was relatively high. Moreover, the BMI of the patients in this study was relatively low, since lower BMI is one of the features of Korean PCOS patients [16]. These factors might affect pregnancy rates and might be responsible for side effects of PCOS such as OHSS, which was observed in three cases in group $A$ and two cases in group $B$, and multiple pregnancy rates, which were $18.4 \%$ in group $A$ and $20.0 \%$ in group $B$. However, adjusting the $\mathrm{hMG}$ dose can control these complications and reduce the cost of COS. Finally, we did not check the effects of CC on the characteristics of the cervical mucus.

This study is a preliminary trial for a new CC regimen aimed at overcoming the anti-estrogenic effects of $\mathrm{CC}$ on the endometrium. Pa- tients with a higher dose and shorter duration of CC showed an increased endometrial thickness and higher pregnancy rate. Further investigation should be performed in large populations, including comparison with patients from other countries and using proper randomization to determine the adequate dose and timing of $\mathrm{CC}$, as well as the appropriate dose of hMG, in infertile patients.

\section{Conflict of interest}

No potential conflict of interest relevant to this article was reported.

\section{References}

1. Homburg R. Clomiphene citrate-end of an era? A mini-review. Hum Reprod 2005;20:2043-51.

2. Kousta E, White DM, Franks S. Modern use of clomiphene citrate in induction of ovulation. Hum Reprod Update 1997;3:359-65.

3. Out HJ, Coelingh Bennink HJ. Clomiphene citrate or gonadotrophins for induction of ovulation? Hum Reprod 1998;13:2358-61.

4. Dehbashi S, Parsanezhad ME, Alborzi S, Zarei A. Effect of clomiphene citrate on endometrium thickness and echogenic patterns. Int J Gynaecol Obstet 2003;80:49-53.

5. Haritha S, Rajagopalan G. Follicular growth, endometrial thickness, and serum estradiol levels in spontaneous and clomiphene citrate-induced cycles. Int J Gynaecol Obstet 2003;81:287-92.

6. Nakamura Y, Ono M, Yoshida Y, Sugino N, Ueda K, Kato H. Effects of clomiphene citrate on the endometrial thickness and echogenic pattern of the endometrium. Fertil Steril 1997;67:256-60.

7. Satirapod C, Wingprawat S, Jultanmas R, Rattanasiri S, Jirawatnotai S, Choktanasiri W. Effect of estradiol valerate on endometrium thickness during clomiphene citrate-stimulated ovulation. J Obstet Gynaecol Res 2014;40:96-101.

8. Esmailzadeh S, Faramarzi M. Endometrial thickness and pregnancy outcome after intrauterine insemination. Fertil Steril 2007;88:432-7.

9. El-Toukhy T, Coomarasamy A, Khairy M, Sunkara K, Seed P, Khalaf $Y$, et al. The relationship between endometrial thickness and outcome of medicated frozen embryo replacement cycles. Fertil Steril 2008;89:832-9.

10. Unfer V, Casini ML, Costabile L, Mignosa M, Gerli S, Di Renzo GC. High dose of phytoestrogens can reverse the antiestrogenic effects of clomiphene citrate on the endometrium in patients undergoing intrauterine insemination: a randomized trial. J Soc Gynecol Investig 2004;11:323-8.

11. Shimoya K, Tomiyama T, Hashimoto K, Moriyama A, Kawamoto A, Tokugawa $Y$, et al. Endometrial development was improved by transdermal estradiol in patients treated with clomiphene ci- 
trate. Gynecol Obstet Invest 1999;47:251-4.

12. Mitwally MF, Casper RF. Aromatase inhibitors in ovulation induction. Semin Reprod Med 2004;22:61-78.

13. Barroso G, Menocal G, Felix H, Rojas-Ruiz JC, Arslan M, Oehninger S. Comparison of the efficacy of the aromatase inhibitor letrozole and clomiphene citrate as adjuvants to recombinant follicle-stimulating hormone in controlled ovarian hyperstimulation: a prospective, randomized, blinded clinical trial. Fertil Steril 2006;86:1428-31.

14. Takasaki A, Tamura H, Taketani T, Shimamura K, Morioka H, Sugi- no N. A pilot study to prevent a thin endometrium in patients undergoing clomiphene citrate treatment. J Ovarian Res 2013; 6:94.

15. Mikkelson TJ, Kroboth PD, Cameron WJ, Dittert LW, Chungi V, Manberg PJ. Single-dose pharmacokinetics of clomiphene citrate in normal volunteers. Fertil Steril 1986;46:392-6.

16. Chae SJ, Kim JJ, Choi YM, Hwang KR, Jee BC, Ku SY, et al. Clinical and biochemical characteristics of polycystic ovary syndrome in Korean women. Hum Reprod 2008;23:1924-31. 